\title{
EFFECT OF DIELECTRIC LOSS ON THE QUALITY FACTORS OF PIEZOELECTRICALLY DRIVEN LENGTH EXTENSIONAL MODE RESONATORS
}

\author{
A. Qamar ${ }^{1}$, S. Sherrit ${ }^{2}$, X.-Q. Zheng ${ }^{3}$, J. Lee ${ }^{3}$, P. X.-L. Feng ${ }^{3}$, and M. Rais-Zadeh ${ }^{1,2}$ \\ ${ }^{1}$ Electrical Engineering and Computer Science, University of Michigan, Ann Arbor, MI, USA \\ ${ }^{2}$ Jet Propulsion Laboratory, California Institute of Technology, Pasadena, CA, USA \\ ${ }^{3}$ Electrical Engineering, Case School of Engineering, Case Western Reserve University, Cleveland, OH, USA
}

\begin{abstract}
In this paper, we present the effect of dielectric loss on quality factors of piezoelectrically actuated length extensional (LE) mode resonators. LE mode resonators having same lateral dimensions but different layer stacking structures, namely silicon ( $\mathrm{Si})$, aluminum/aluminum nitride (Al/AlN), $\mathrm{AlN} / \mathrm{Si}$ and $\mathrm{Al} / \mathrm{AlN} / \mathrm{Si}$, are designed and fabricated. The corresponding resonance and quality factors $(Q s)$ are measured using optical and electrical readout techniques. The effect of different loss mechanisms in each layer on the $Q \mathrm{~s}$ is investigated by methodically removing each layer in simulation and fabrication. Thermoelastic damping (TED) in an assumed homogenous AIN film and anchor loss have been investigated by COMSOL, and the dielectric loss or loss tangent in the piezoelectric layer has been modelled by using the Mason's network model. The effect of AlN dielectric loss tangent on measured $Q$ values is analyzed using Mason's model, and its dependence on piezoelectric loss is discussed.
\end{abstract}

\section{INTRODUCTION}

Piezoelectric thin films have been investigated since the early developments of MEMS resonator technology. Zinc oxide $(\mathrm{ZnO})$, lead zirconate titanate (PZT), lithium niobate $\left(\mathrm{LiNbO}_{3}\right)$, and $\mathrm{AlN}$ are the main piezoelectric thin film materials in MEMS industry today. AlN is much suitable choice for many RF applications due to its fabrication simplicity and reproducibility as well as its low dielectric loss [1], minimal electronic contamination issue, and high thermal conductivity [2]. Piezoelectrically actuated resonators may utilize high-quality single-crystal $\mathrm{Si}$ as the structural layer and a thin film piezoelectric only as the transduction layer [3]. However, the presence of the piezoelectric layer degrades the $Q$ considerably, a phenomenon, which is not completely understood up to now. Different loss mechanisms including anchor loss [4], TED in the metal electrodes [5], and charge redistribution loss [6,7], have been investigated as possible dominant loss mechanisms in different types of piezoelectric resonators. Among a broad spectrum of losses including mechanical, dielectric and piezoelectric [8], dielectric losses have not been comprehensively considered in piezoelectrically actuated resonators. This loss mechanism does not affect the thickness-mode resonators (due to the presence of a negative capacitance in its effective model). In length extensional (LE) mode resonators that have lower $f \times Q$ values, dielectric loss is a factor we explore here in detail.

In this study, we analyzed the loss tangent using the Mason's network model for lateral length extensional resonators, along with the network model of an elastic layer vibrating along its length. The composite resonator consists of different heterostructures of thin film materials coalesced at their interfaces. Using the extended model, the effect of dielectric loss in piezoelectric layer (AIN) has been analyzed for the first time, with measured value for loss tangent of AlN at the frequency of resonance. In addition to studying the effect of dielectric losses on mechanical $Q$ s of the resonators, we study the effect of all other losses that were proposed as the main $Q$ limiting mechanisms and explain their contributions, which are measured and analyzed to be small and not dominant.

\section{DESIGN AND FABRICATION}

LE mode resonators having a resonance frequency of $\sim 10 \mathrm{MHz}$ are designed for studying their $Q$ values and loss mechanisms. The LE mode was chosen due to the insensitivity of its resonance frequency to the number of layers in the resonance stack, as well as due to its substantially lower measured $Q$ compared to what is expected due to known $Q$ limiting loss mechanisms. COMSOL finite element modeling (FEM) tool was used to assess the exact resonance frequency of each type of resonator with its specific structural stack of constituting layers. Anchor loss using perfectly matched layers (PML) model was used to design the tethers and calculate the anchor loss using COMSOL. TED for each type of stack with and without metal electrodes was analyzed using standard thermoelasticity modelling of COMSOL. Akheizer loss values for Si and AlN layers are taken from [9] and accounted for in total estimated $Q$ values. The simulated $Q$ value for each layer is given in Fig. 1 along with the fundamental resonance mode of LE resonator.

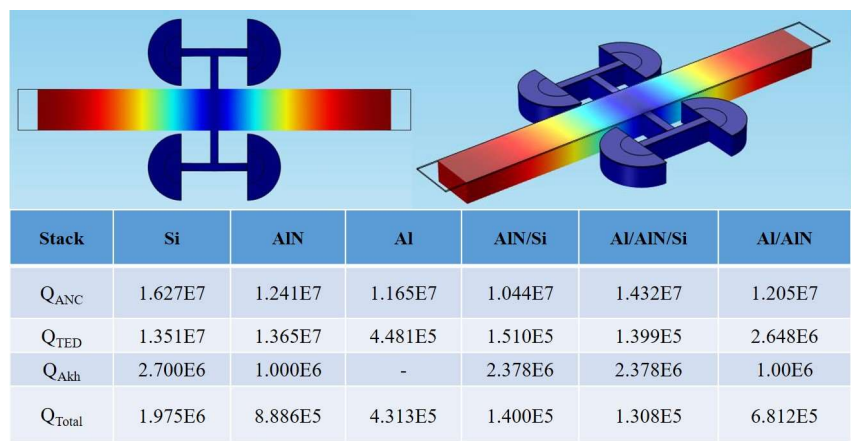

Figure 1: Simulation of the LE resonance mode, with corresponding simulated $Q$ for each type of stacking.

LE mode resonators having different heterostructures stacking were fabricated using standard MEMS processes of photolithography, dry etching and $\mathrm{XeF}_{2}$ releasing as shown in Fig. 2, to study the $Q$ limiting mechanism in this type of resonators. A vector network analyzer (VNA) was used to measure the electrical transmission response of $\mathrm{Al} / \mathrm{AlN} / \mathrm{Si}$ heterostructure resonators, and to calculate the $Q$ values as shown in Fig. 3(a). A custom-built optical in-plane (lateral) displacement measurement system based on knife-edge effect, as shown in Fig. 3 (b), was used to measure the $Q$ of each layer, especially for the stacks without Al electrodes. Figure 3(c) shows the optically measured resonance response and $Q$ of the $\mathrm{Al} / \mathrm{AlN} / \mathrm{Si}$ resonator using the optical measurement system, which agrees well with the electrical measurements.

\section{EXPERIMENTAL RESULTS AND DISCUSSION}

The results for all the remaining resonator stacks without $\mathrm{Al}$ electrodes, measured using optical technique, are shown in Fig. 4. A complete summary of measured and simulated $Q$ s is given in Table 1. It can be observed from Table 1 that TED, which is proposed as the dominant loss mechanism in piezoelectric resonators and is believed to be originating from metal electrode [5], is in fact not the 
dominant loss mechanisms for LE mode resonators. This can be observed by comparing the $Q$ drop from $\mathrm{Si}$ only to $\mathrm{AlN} / \mathrm{Si}$ resonators in Table 1; also comparing simulated $Q$ s of $\mathrm{Al} / \mathrm{AlN} / \mathrm{Si}$ and $\mathrm{Al} / \mathrm{AlN}$ suggest the TED $Q$ should be even higher for $\mathrm{Al} / \mathrm{AlN}$ resonators but that is not the case in experiment. The reason the simulated $Q$ for AlN only and $\mathrm{Al} / \mathrm{AlN}$ resonators is high is due to the very good thermal property of an assumed homogenous AlN film. But in experiment, comparing the $Q$ s of all stacks shows the main lossy layer is the AlN itself and not the metal layer on top of it. Analysis of measured and simulated $Q$ s for each type of resonator also reveals that the anchor loss is not the dominant mechanism in these resonators either (Table 1). Charge redistribution loss cannot explain the very low $Q$ seen in these resonators with thin AlN film acting as the main structural layer. If this mechanism was the main mechanism, the $Q$ of $\mathrm{Al} / \mathrm{AlN} / \mathrm{Si}$ should have been about half of $\mathrm{AlN} / \mathrm{Si}$ resonators but the drop of measured $Q$ when adding $\mathrm{Al}$ is insignificant compared to the drop seen when adding AlN to the starting Si layer.
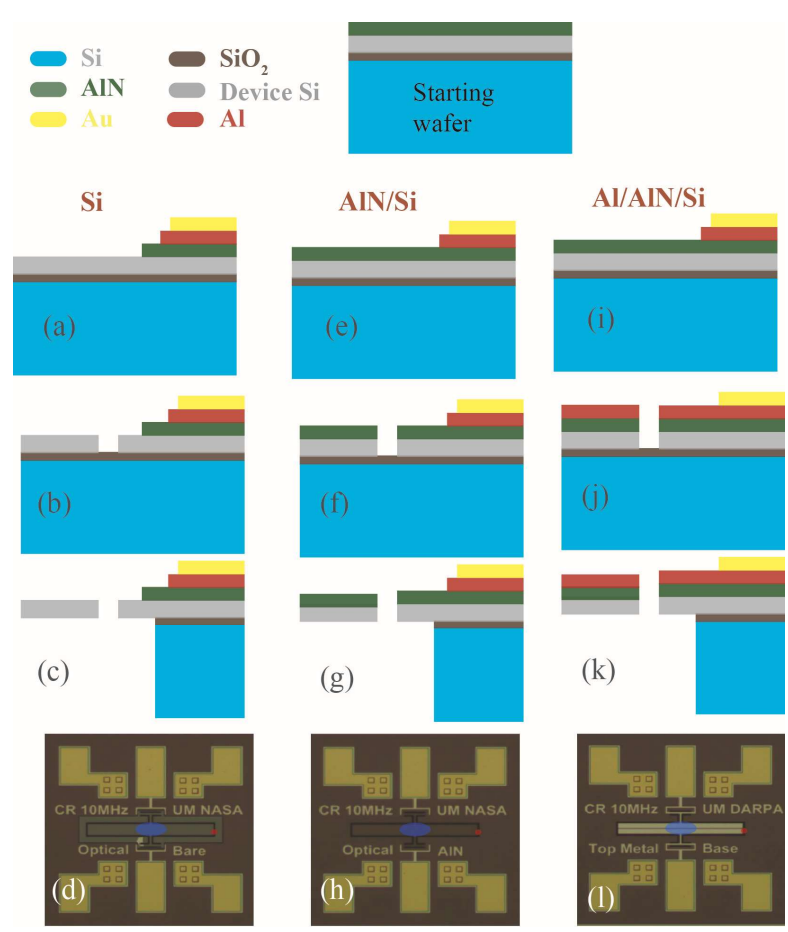

Figure 2: Device structure and fabrication process with starting SOI wafer, (a)-(c) Si resonator, (e)-(g) Al/Si resonator, (i)-(k) Al/AlN/Si resonator, (d),(h),(l) fabricated device images. DRIE was used for backside etching and $\mathrm{XeF}_{2}$ release was used for front side release of $\mathrm{Si}$ device layer. Al/AlN resonators were fabricated by releasing the Si device layer of Al/AIN/Si resonator in $\mathrm{XeF}_{2}$.

Let us summarize the effect of each of the known charge mechanisms: 1) TED loss in the metal layer, as proposed in [5] to be the dominant mechanism, is found experimentally here to be not significant. Comparing the measured $Q$ of $\mathrm{Si}$ only, $\mathrm{AlN} / \mathrm{Si}$, and $\mathrm{Al} / \mathrm{AlN} / \mathrm{Si}$ resonators shows that the main drop in $Q$ is observed when AlN is added to the Si only stack ( $Q$ of Si only resonator is $>300,000$, whereas $Q$ of the AlN/Si device is only 27,800 and $Q$ of the $\mathrm{Al} / \mathrm{AlN} / \mathrm{Si}$ resonator $(\sim 22,000)$ is very similar in value to that of the AIN/Si resonator). Anchor loss is simulated to be at least an order of magnitude larger than the measured $Q$ for all resonator stacks. Charge redistribution loss should reduce the $Q$ of $\mathrm{Al} / \mathrm{AlN} / \mathrm{Si}$ resonator to half the value of $\mathrm{AlN} / \mathrm{Si}$ resonator but that is not the case (in fact the $Q$ s of these two resonators is found to be quite close and only $20 \%$ different).

As TED, anchor loss, and charge redistribution loss are unable to explain the observed drop of $Q$ in LE mode resonator, the loss tangent was next investigated by using the Mason's network model. We have used Mason's network model to investigate the effect of loss tangent, because COMSOL default simulation does not account for such losses in the dielectrics. Additionally, Mason's network model provides analysis of piezoelectric loss and any intrinsic loss separately; and their interdependence on each other as discussed in the next section.

Table 1: Simulated and measured $Q$ in million for resonators with different stack materials. Simulated $Q$ only accounts for TED, anchor loss, and Akheizer loss in Si and AlN layers (Fig. 1).

\begin{tabular}{|l|l|l|l|l|l|l|}
\hline & $\mathbf{S i}$ & $\mathbf{A l N}$ & $\mathbf{A l}$ & $\mathbf{A l N} / \mathbf{S i}$ & $\mathbf{A l} / \mathbf{A l N} / \mathbf{S i}$ & $\mathbf{A l} / \mathbf{A l N}$ \\
\hline $\begin{array}{l}Q \\
\text { Simulated }\end{array}$ & 1.97 & 0.88 & 0.43 & 0.14 & 0.13 & 0.68 \\
\hline $\begin{array}{c}Q \\
\text { Measured }\end{array}$ & 0.30 & - & - & 0.0278 & 0.022 & $\mathbf{0 . 0 0 2 4}$ \\
\hline
\end{tabular}
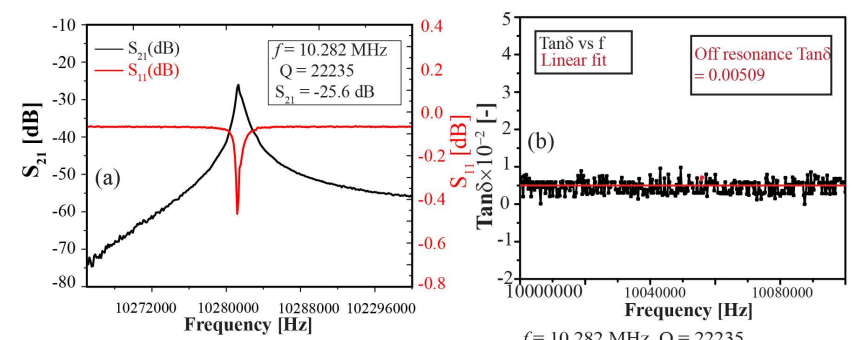

(c)

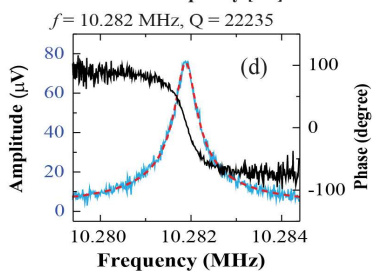

Figure 3: (a) Al/AlN/Si electrical transmission measurements along with the measured $Q$ value. (b) Measurement of tan $\delta$ in AlN using impedance data. (c) Optical measurement illustration. (d) Optically measured resonance of Al/AlN/Si resonator.
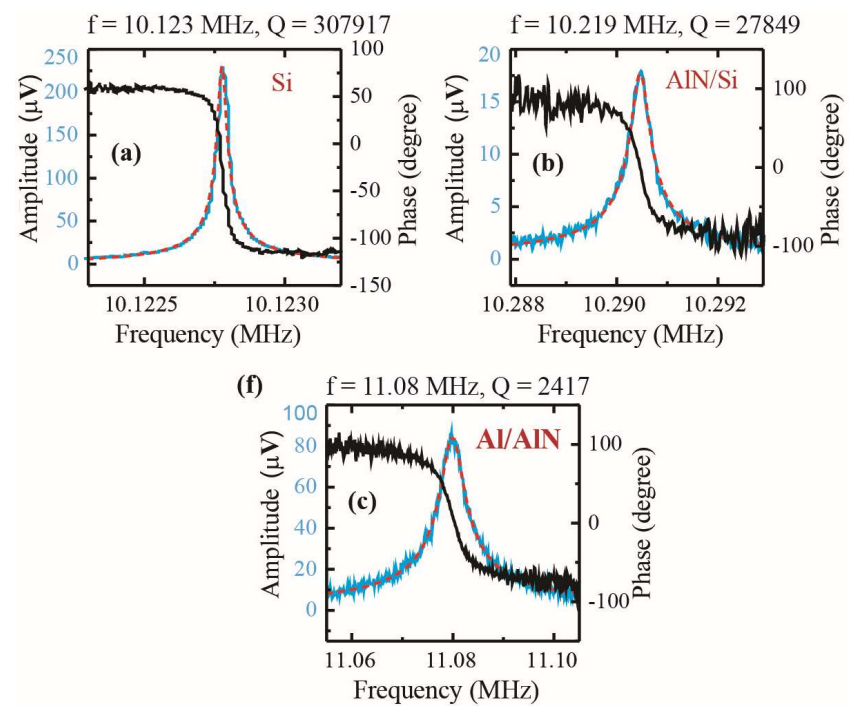

Figure 4: Optical measurement results for each type of resonator labelled on the plot. 


\section{LOSS TANGENT BY MASON'S MODELLING}

In order to model piezoelectric transducers, different 1D modelling techniques were used before the development of FEM commercial software packages, such as COMSOL. It is quite difficult to find analytical solutions to the wave equation in piezoelectric materials using first principles. Mason [10] showed that network theory can be used for one-dimensional analysis to overcome most of the difficulties in deriving the solutions. He presented an exact equivalent circuit that separated the piezoelectric material into an electrical port and two acoustic ports with an ideal electromechanical transformer for a length thickness resonator geometry. Berlincourt et al. investigated a variety of modes [11] and Sherrit et al. [12] extended the models to include losses in all the material constants to account for various damping mechanisms. In an effort to remove circuit elements between the top of the transformer and the node of the acoustic transmission line, Krimholtz, Leedom and Matthae [13] published an alternative equivalent circuit which was found to produce identical results [12].

The model we used to fit the optical experimental data is based on Mason's network equivalent for a piezoelectric resonator and is shown in Fig. 5. In the mechanical network, forces act like voltages and currents, represent the velocity of a face of the resonator. The model below represents three layers ( $\mathrm{Si}, \mathrm{AlN}$ and $\mathrm{Al}$ of $20 \mu \mathrm{m}, 1$ $\mu m, 0.1 \mu m$, respectively). The force is applied via laser excitation, which causes the resonator bar to vibrate with a velocity $v$. The velocity of the free face $v$ can be integrated to get the displacement amplitude $(d=v / i \omega)$, where $\omega$ is the frequency of the excitation. During the resonance the strain, displacement and velocity are considered the same. This data can then be fit to the optical measurements and material constants in the model can be adjusted to look at the effect of the elastic, dielectric and piezoelectric loss. The elastic, dielectric, and piezoelectric constants of the model are treated as complex quantities with the imaginary component representing the loss. The functions $Z_{T}$ and $Z_{S}$ are based on the tan and secant functions with the frequency, density, length, area, and acoustic velocity of the layers. $N$ is the turn's ratio for the piezoelectric transformer and the $Z_{o}$ term represents the static capacitance of the piezoelectric layer. The elastic constants can then be written as

$$
Y=Y_{r}+i Y_{i}=Y_{o}(1-i 1 / Q) .
$$

While the piezoelectric and dielectric constant can be written as:

$$
\begin{aligned}
& d_{31}=d_{31 r}+i d_{31 i} \\
& \varepsilon_{31}=\varepsilon_{31 r}+i \varepsilon_{31 i}
\end{aligned}
$$

An example of the fit to the $\mathrm{Si} / \mathrm{AlN} / \mathrm{Al}$ and $\mathrm{Al} / \mathrm{AlN}$ optical data is shown in Fig. 6 using measured value for loss tangent of AlN (as shown in Fig. 3). The baseline material properties used for each layer of the resonator are shown in Table 2. In each of the resonator models, we had to adjust the Young's modulus of the AlN layer to account for changes in the layer boundary conditions and its effect on the acoustic velocity in the layer, to match the resonance peak. In addition, a slight modification in the real part of the elastic constant was required to adjust for the different piezoelectric coefficients. The mechanical $Q$ of the Si layer was fixed at 307,91 as determined experimentally from the bare $\mathrm{Si}$ resonator. The $Q$ of the $\mathrm{Al}$ was fixed at 60,000 (a large enough value not to be the dominant effect to allow studying the effect of dielectric loss in AlN only). The imaginary part of the permittivity (tan $\delta$ ) was adjusted according to measured value from impedance data (Fig. 3d) to reproduce the experimental data of optically measured 2 and 3 layer stacked devices, using 3 different values of the piezoelectric coefficient $\left(d_{31}=-2.1 p C / N,-1.1 p C / N\right.$ and complex $\mathrm{d}_{31}=(-1.1$ $0.0223 i) p C / N$ ). The results for the analysis are shown in Figure 6.

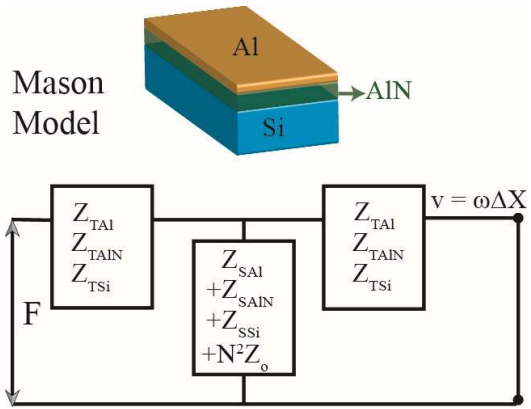

Figure 5: The electromechanical network equivalent of three-layer resonator vibrating in the LE mode based on Mason's network equivalent $[8,9]$. The electrical impedance of the piezoelectric $Z_{o}$ has been transformed to the acoustic elements and represents a dissipation that can reduce the effective $Q$ of the resonator.
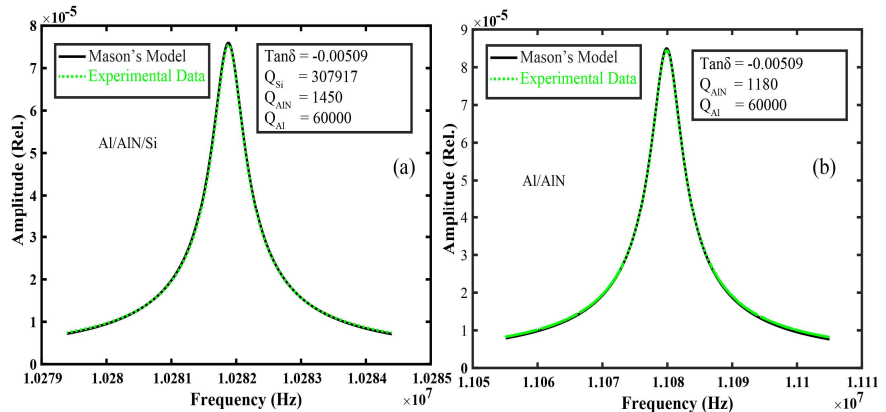

Figure 6: The optical resonance data and a fit using the model shown in Figure 5 for (a) The Al/AlN/Si stack and (b) Al/AlN layers.

Table 2: The real component of the material properties of each material in the resonator stack.

\begin{tabular}{|c|c|c|c|c|}
\hline Material & $\mathbf{S i}$ & $\mathbf{A l}$ & $\begin{array}{c}\mathbf{A I N} \text { in } \\
\mathbf{A I N} / \mathbf{S i}\end{array}$ & $\begin{array}{c}\mathbf{A I N} \text { in } \\
\mathbf{A l} / \mathbf{A l N} / \mathbf{S i}\end{array}$ \\
\hline $\begin{array}{c}\text { Density } \\
\left(\mathrm{kg} / \mathrm{m}^{3}\right)\end{array}$ & 2328 & 2700 & 3260 & 3260 \\
\hline $\begin{array}{c}\text { Y Elastic } \\
\text { Constant } \\
\left(\mathrm{N} / \mathrm{m}^{2}\right)\end{array}$ & $\begin{array}{c}1.69 \\
\times 10^{11}\end{array}$ & $\begin{array}{c}0.69 \\
\times 10^{11}\end{array}$ & $\begin{array}{c}3.3199 \times \\
10^{11}\end{array}$ & $\begin{array}{c}2.7549 \times \\
10^{11}\end{array}$ \\
\hline $\begin{array}{c}\varepsilon \\
\text { Permittivity } \\
(\mathrm{F} / \mathrm{m})\end{array}$ & - & - & $\begin{array}{c}1.434 \times 10^{-} \\
10\end{array}$ & $\begin{array}{c}1.434 \times 10^{-} \\
10\end{array}$ \\
\hline $\begin{array}{c}d_{31} \\
\text { Piezoelectric } \\
\text { Constant } \\
(\mathrm{pC} / \mathrm{N})\end{array}$ & - & - & -1.1 to -2.1 & -1.1 to -2.1 \\
\hline
\end{tabular}

The data in Fig. 7 show the required effective $Q$ of the AlN layer applied to the model to match the optical data with varying the electrical $\tan \delta$. The data in Figure 7 and the data in Table 3 are modeled to produce optical resonance curves that match the optical data in Table 3. For example, for a given $d_{31}=-1.1 p C / N$ and no piezoelectric loss on the $\mathrm{Al} / \mathrm{AlN} / \mathrm{Si}$ resonator, the measured $\tan \delta$ of 0.00509 suggests that the mechanical $Q$ of the AlN needs to be of the order of 1450 to model the curve in Figure 6a. However, if the 
piezoelectric coefficient was $2.1 \mathrm{pC} / \mathrm{N}$, a $\tan \delta$ of 0.07 would require a mechanical $Q$ in the AIN film to be 10,000 . The measured dielectric loss $(\tan \delta)$ of these films, measured slightly off resonance suggests that mechanical $Q$ of the films is the limiting factor in the overall $Q$ of the resonator. One thing which is evident is that the larger the piezoelectric coefficient, the smaller the required dielectric loss to account for the $Q$ of the experimental data as shown in Fig. 7. The sign of the piezoelectric loss is also seen to be a factor to have influence on the measured $Q$ values. The more negative the piezoelectric loss, the smaller the electrical $\tan \delta$ required to account for the $Q$ of the resonator.

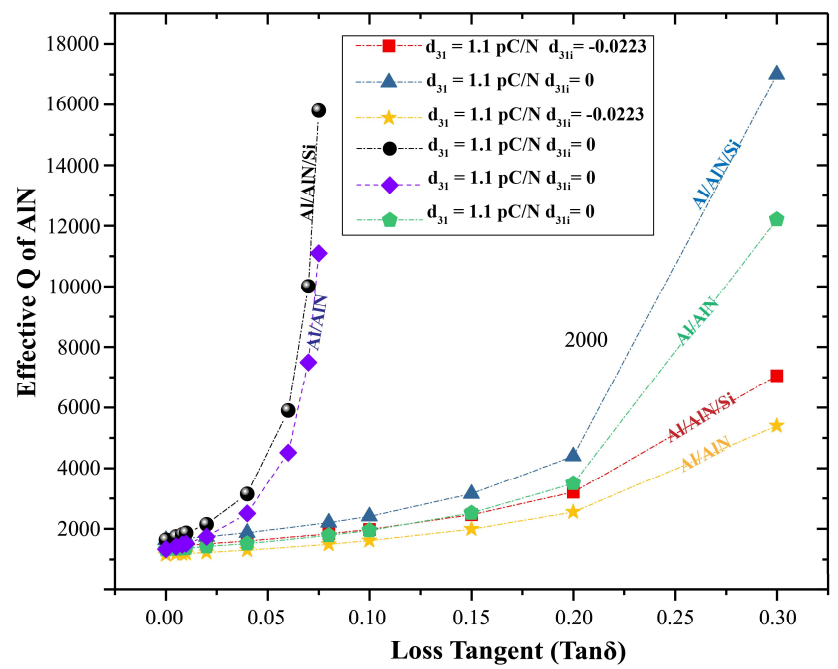

Figure 7: The required $Q$ of the AlN resonator as a function of the tan $\delta$ of the piezoelectric AlN to match the optical data for the 3 layers of Al/AlN/Si and 2 layers of Al/AlN. Here, Tan $\delta_{d}=d_{31 i} / d_{31}$ is the piezoelectric loss tangent.

Different possible scenarios for the $Q$ of each layer and the composite resonators are given in Table 3. It can be observed from Table 3 that the experimentally observed value of $Q$ for $\mathrm{Al} / \mathrm{AlN}$ and $\mathrm{Al} / \mathrm{AlN} / \mathrm{Si}$ resonators can be explained if the dielectric loss values were high (the low value of 0.005 shows the dielectric loss is the not dominant $Q$ limiting mechanism either). In addition, the low $Q$ can only be explained only if AlN layer has a high mechanical loss that is not considered in conventional TED, anchor loss, and surface loss models [14]. Si layer is known to have a high $Q$ as extracted from the measured $Q$ using optical technique.

Table 3: Mason Model estimation of tan $\delta$ and other internal losses within each material to match the measured $Q$ for AlN/Al (last column) and Al/AlN/Si resonators. Measured value of loss tangent is 0.005 which means the contribution of dielectric loss in AlN in not significant and some other loss in AlN needs to be high to match the measured $Q$.

\begin{tabular}{|l|l|l|l|}
\hline $\tan \boldsymbol{\delta}$ & $\boldsymbol{Q}$ mechanical: $\mathbf{A l}$ & $\boldsymbol{Q}$ mechanical: $\mathbf{A I N}$ & $\boldsymbol{Q}$ total \\
\hline 0.0003 & 2474 & 2474 & 2474 \\
\hline 0.022 & 6000 & 3000 & 2474 \\
\hline 0.034 & 60000 & 3000 & 2474 \\
\hline 0.042 & 60000 & 60000 & 2474 \\
\hline 0.0050 & 60000 & 2474 & 2474 \\
\hline
\end{tabular}

\section{CONCLUSION}

Effect of dielectric loss on the quality factors of piezoelectrically driven LE mode resonators is investigated. The effect of different heterostructure stacking on the $Q$ s is analyzed by
COMSOL simulations and then by experimentally measuring the $Q$ s using electrical and optical readout techniques. Mason's model together with the experimental data is used to investigate the effect of dielectric loss in AlN thin film. It has been found that the AlN has low dielectric loss and the mechanical $Q$ of AIN is responsible for low $Q$ s in the LE mode resonators.

\section{ACKNOWLEDGEMENT}

This work is supported by Army Research Office (ARO), the Defense Advanced Research Project Agency (DARPA), and by NASA.

\section{CONTACT}

*A. Qamar, tel: +1-(734) 730-8083; afzaal@umich.edu

*M. Rais-Zadeh, tel: +1-(734) 764-4249; $\underline{\text { minar@umich.edu }}$

\section{REFERENCES}

[1]. E. Savrun et al., "High thermal conductivity aluminum nitride ceramics for high power microwave windows," Fifth IEEE International Vacuum Electronics Conference, (IEEE Cat. No.04EX786) 2004, pp. 45-46.

[2]. J. C. Doll et al., "Aluminum nitride on titanium for CMOS compatible piezoelectric transducers," Journal of micromechanics and microengineering: structures, devices, and systems, 20, 25008 (2010).

[3]. A. Jaakkola, et al., "Piezoelectrically transduced singlecrystal-silicon plate resonators," IEEE Ultrason Symp, Beijing (2008), pp. 717-720

[4]. J. Segovia-Fernandez et al., "Anchor losses in AlN contour mode resonators," JMEMS, 24, 265 (2015).

[5]. J. Segovia-Fernandez et al., "Thermoelastic damping in the electrodes determines Q of AlN contour mode resonators," JMEMS, 26, 550 (2017).

[6]. A. Peczalski, et al., "Effects of heterostructure stacking on acoustic dissipation in coupled-ring resonators," IEEE MEMS, Las Vegas (2017), pp. 954-957.

[7]. R. Tabrizian et al., "The effect of charge redistribution on limiting the kt2.Q product of piezoelectrically transduced resonators, "Transducers-18th International Conference on Solid-State Sensors, Actuators and Microsystems, Anchorage (2015), pp. 981-984.

[8]. A. V. Mezheritsky, "Elastic, dielectric, and piezoelectric losses in piezoceramics: how it works all together," in IEEE Transactions on Ultrasonics, Ferroelectrics, and Frequency Control, 51, 695 (2004).

[9]. R. Tabrizian et al., "Effect of phonon interactions on limiting the f.Q product of micromechanical resonators," TransducersInternational Solid-State Sensors, Actuators and Microsystems Conference, Denver (2009), pp. 2131-2134.

[10]. W.P. Mason, Electromechanical Transducers and Wave Filters, Princeton, NJ, Van Nostrand, 1948.

[11]. D.A. Berlincourt et al., Academic Press, W.P. Mason- editor pp. 169-270.

[12]. S. Sherrit et al., "Comparison of the Mason and KLM equivalent circuits for piezoelectric resonators in the thickness mode," Proceedings of the IEEE Ultrasonics Symposium, Lake Tahoe (1999), pp. 921-926.

[13]. R. Krimholtz et al., "New equivalent circuits for elementary piezoelectric transducers," Electronics Letters, 6, (398) 1970.

[14]. Explaining the anomalous low quality factors in piezoelectrically driven length extensional mode thin Film resonators (under review). 